\title{
Bilayer Distributed Energy Model with Pollution Consideration
}

\author{
Lin Yang*, Ming Li, Xiangchao Gong, Desan Ji, Mingjin Liao
}

College of Science, Wuhan University of Science and Technology, Wuhan, China

\section{Email address:}

Yanglin830521@gmail.com (Lin Yang), mingli121212@126.com (Ming Li), sunray9343@126.com (Xiangchao Gong), gxc741@163.com (Desan Ji), wazjn2006@163.com (Mingjin Liao)

${ }^{*}$ Corresponding author

\section{To cite this article:}

Lin Yang, Ming Li, Xiangchao Gong, Desan Ji, Mingjin Liao. Bilayer Distributed Energy Model with Pollution Consideration. Science Discovery. Vol. 4, No. 2, 2016, pp. 79-85. doi: 10.11648/j.sd.20160402.15

Received: March 17, 2016; Accepted: April 14, 2016; Published: May 4, 2016

\begin{abstract}
In this paper, the use of energy resource is combined with environmental pollution to establish the model of distributed energy system. The pollution problem is reasonally assessed by using environomics as the optimization tool of energy system, considering environment as consumable resources, and quantitative analysis on the environment problem caused by pollutants. The optimal solution is given by use of genetic algorithm with the comprehensive cost which contains pollution processing costs of energy supplying point and fines for pollution as the objective function. Besides, the network layout of supply chain of distributed energy system is also discussed with emphasis. The supply chains and heat supply pipelines of the whole distributed energy system are reasonally designed with a double programming model. Finally, the energy system is comprehensively assessed under the uncertain condition and unsafe factors, and then is optimized with a robust method, so that the model of distributed energy is economical, reasonal, safe and credible.
\end{abstract}

Keywords: Distributed Energy System, Pollution, Environomics

\section{考虑污染因素的双层分布式能量模型}

\author{
杨琳 ${ }^{*}$, 李明, 龚向超, 吉德三, 廖明进 \\ 理学院力学系, 武汉科技大学, 武汉, 中国 \\ 邮箱 \\ Yanglin83521@gmail.com (杨琳), mingli121212@126. com(李明), sunray9343@126. com（龚向超）, gxc741@163. com（吉德三)， \\ wazjn2006@163. com（廖明进）
}

\begin{abstract}
摘要: 本文将能源的使用与环境污染问题结合, 建立分布式能量系统的模型。以㶲经济学作为能量系统的优化工具, 环境为可消耗的资源，把污染物引起的环境问题作量化分析，对污染进行合理评估。以供应点包含污染处理费用、污 染罚款在内的综合费用为目标函数, 使用遗传算法求解最优解。同时, 重点讨论了分布式能量系统供应链的网络规划, 使用双层规划模型对整个分布式能量系统的供应点及输热管道进行合理规划, 并对此能源系统在不确定条件和不安全 因素下进行综合评估，使用鲁棒法进行优化，以得出经济、合理、安全、可靠的分布式能源模型。
\end{abstract}

关键词: 分布式能量系统, 污染, 㶲经济学 


\section{1. 引言}

能源是人类生存和发展的重要物质基础, 也是当今世 界政治、经济、军事、外交关注的焦点 [1]。随着经济的 发展, 人类对能源的需求急剧增加。然而对非可再生能源 过度利用, 又使得能源储量急剧减少, 导致世界能源的生 产和环境的承载能力不堪重负。环境污染问题变得越来越 严重 [2]。传统的集中式供能系统特点是大型化和集中化, 其网络分布比较复杂, 并且用户多集中于某一的区域内, 这样其在适应负荷变化的灵活性和供电安全性方面就存 在一些弊端 [3]。传统的能源供应方式主要是天然气或电 直接供热、燃煤供暖与集中式热电并供, 这些供能方式存 在着一些明显不足, 比如环境污染、电力与天然气的峰谷 之差加大、能源浪费、管道网络投资巨大等 [4]。而分布 式能量系统提出, 在很大程度上解决这些问题 [4-6]。目 前对分布式能量系统的研究中, 大多是将能量高效利用作 为模型优良的衡量标准, 很少考虑能量系统在运营时所产 生的污染问题, 因此本文在考虑建立优化的双层能量模型 的同时将污染参数加入模型中, 这对当前的环境建设更有 深远的意义。

\section{2. 建立模型}

充分考虑污染因素、成本、产能相关费用及管道铺设 费用等, 对分布式能量系统的供应点布置和管道规划进行 分析, 以分布式能量系统的总费用为目标函数, 建立双层 规划模型。

以年为时间周期, 将输热管道的固定投资及维修的费 用平均分推到能源系统整个生命周期的每一年内, 由于不 同时间的能量需求不同，故将一年内客户的需求量分为春 夏秋冬四个不同阶段, 将每个阶段中的一天又划分为需求 高峰期和普通期两个阶段, 此阶段内时间单位为小时。为 简化模型, 提出以下假设:

1) 每一客户只归属于一个供应点。2) 用户的电能和热 能需求均可由此系统中的能源供应点满足。3) 热能输送可 以通过某一客户后再到达下一客户, 忽略输热管在输送热 能时的能量损失。4) 不考虑管道容量的限制, 假设一根管 道可途经多个客户并能提供客户所需要所有的热能。

\section{1. 数学模型建立} 型:

根据分布式能量系统的费用组成, 可建立以下数学模

$$
\begin{aligned}
\text { Min } \quad C_{\text {total }} & =c_{s c r} \sum_{j \in S} C_{f 0}+\sum_{t \in T} c_{v f} s h m_{j} \\
& +c_{o h} \sum_{t \in T} \sum_{j \in S} s h_{j}^{t}+c_{o p} \sum_{t \in T} \sum_{j \in S} s p_{j}^{t}+C_{p}+C_{S L}
\end{aligned}
$$

其中等式左边 $C_{\text {total }}$ 表示年均能源供应成本, 右边第 一项 $c_{s c r} \sum_{j \in S} C_{f 0}$ 表示供应点的成本, $c_{s c r}$ 为污染处理费用
扩大系数, 为右边第二项 $c_{v f} s h m_{j}$ 表示与产能相关的供应 点建设固定成本, $c_{v f}$ 为其系数, 右边第三项 $c_{o h} \sum_{t \in T} \sum_{j \in S} s h_{j}^{t}$ 表示热能生产成本, $c_{o h}$ 为单位热能的成本, $s h_{j}^{t}$ 为热能生 产量, 右边第四项表示电能生产成本, $c_{o p}$ 为单位电能成 本, $s p_{j}^{t}$ 为电能生产量, 右边第五项 $C_{p}$ 表示污染罚款, 此 项值的大小与第一项中 $c_{s c r}$ 及 $s h m_{j}$ 密切相关。右边第六项 $C_{S L}$ 表示输热管道成本。 $S$ 表示供应点的集合, $T$ 表示各 时间阶段的集合。

定义用户集合为 $C$, 对于 $i \in C$, 若 $i$ 归属于供应点 $j$, 则令 $X_{i j}=1$, 否则令 $X_{i j}=0$ 。此模型约束条件有:

1) 供应点能供需守恒:

$$
\begin{gathered}
s h_{j}^{t}=\sum_{i \in C} X_{i j} d h_{i}^{t}, \forall j \in S, \forall t \in T \\
\sum_{j \in S} s h_{j}^{t}=\sum_{i \in C} d h_{i}^{t}, \forall t \in T \\
s p_{j}^{t}=\sum_{i \in C} X_{i j} d p_{i}^{t}, \forall j \in S, \forall t \in T \\
\sum_{j \in S} s p_{j}^{t}=\sum_{i \in C} d p_{i}^{t}, \forall t \in T
\end{gathered}
$$

其中 $d h_{i}^{t} 、 d p_{i}^{t}$ 分别表示客户 $i$ 在 $t$ 时间段的热能能和 电能需求量。

2) 最大需求及供应:

$$
\begin{gathered}
s h m_{j}=\max \left\{s h_{j}^{t} \mid t=1,2,3, \ldots, T\right\}, \forall j \in S \\
s p m_{j}=\max \left\{s p_{j}^{t} \mid t=1,2,3, \ldots, T\right\}, \forall j \in S \\
d h m_{i}=\max \left\{d h_{i}^{t} \mid t=1,2,3, \ldots, T\right\}, \forall i \in C
\end{gathered}
$$

其中 $s h m_{j} 、 s p m_{j} 、 d h m_{i}$ 分别表示供应点 $j$ 的热能、 电能的产能和客户最大热能需求量。

$C_{S L}$ 为下层规划目标函数, 由以下规划确定:

$$
\operatorname{Min} \quad C_{S L}=\sum_{j \in S} \sum_{l_{j} \in L_{j}} c_{d} d_{l_{j}}
$$

其中, 等式左边为输热管道成本, 等式右边 $L_{j}$ 为以 供应点 $j$ 为起点的管道的集合, $l_{j}$ 为其中的元素, $c_{d}$ 表示 单位长度的管道的成本, $d_{l_{j}}$ 表示输热管道 $l_{j}$ 的长度。

定义 $Y_{i k l_{j}} \in\{0,1\}$, 若 $i$ 到 $k$ 之间有输热管, 则 $Y_{i k l_{j}}=1$, 否则 $Y_{i k l_{j}}=0$, 有如下约束条件:

1) 管道的唯一性: 


$$
\sum_{l_{j} \in L_{j}} \sum_{i \in C_{o}} Y_{i k l_{j}}=1, \forall k \in C
$$

2) 热能的守恒:

$$
\sum_{l_{j} \in L_{j}} \sum_{i \in C_{o}} y_{i k l_{j}}-d h m_{k}=\sum_{l_{j} \in L_{j}} \sum_{i \in C} y_{k m l_{j}}, \forall k \in C
$$

其中 $y_{i k l_{j}}$ 表示从节点 $i$ 直接到节点 $k$ 时管道 $l_{j}$ 中剩余 热量。 $C_{o}$ 表示包括客户和供应点在内的所节点集合。

3) 管道长度条件:

$$
d_{l_{j}}=\sum_{i \in C_{o}} \sum_{k \in C} d_{i k} X_{i j} Y_{i k l_{j}}, \forall j \in S
$$

式中的 $X_{i j}$ 和 $d h m_{k}$ 可由上层规划来确定。

\section{2. 模型的求解}

\subsection{1. 求解思路}

此双层规划模型分为上层规划和下层规划两个部分, 上层规划描述为在满足客户电能和热能需求的条件下, 确 定分布式能量系统供应站的数量、坐标以及产能, 使得总
成本最小; 下层规划描述为当分布式能量系统的供应点坐 标, 客户坐标、客户需求量以及客户归属确定之后, 如何 规划输热管道, 能使得热能输送的成本最小。

双层规划的求解是一个难点, P. Hansen, G. Savard 和B. Jaumard用严格和复杂的理论证明了双层线性规划问 题是强NP-hard问题 [7], 对于非线性的双层规划问题, 求 解的难度更加大 [8]。因此, 对于上述双层规划模型, 无 法算出严格的最优解, 只能通过某些特定的求解和优化算 法, 计算出模型的较优解。

以 $\mathrm{k}$ 表示供应点的数量, 首先要根据上层规划估计一 个较优的 $\mathrm{k}$ 值, 这一步可以通过粗略的成本值与 $\mathrm{k}$ 的函数关 系, 通过求极值估算得到。其次, 需要根据客户的坐标, 确定供应点的坐标以及客户的归属, 由模型可得: 供应点 应布置在归属于它的所有客户的坐标的中心位置, 因此, 采用 $\mathrm{k}$-means 聚类算法来求解客户的归属及供应点的位置。 使用 $K$-means算法可以得到较理想的解。而对于输热管道 的铺设, 也是一个非常复杂的问题, 涉及到图论的相关知 识, 属于图论中的最短路径问题。因此选择用最小生成树 相关方法求解。

使用MATLAB软件, 首先随机生成各客户的坐标与需求 量, 再编写相关程序求解并优化。以 $K=2, n_{c}=20$ 为例, 使用 $\mathrm{k}$-means算法得到的结果见图1及图2。

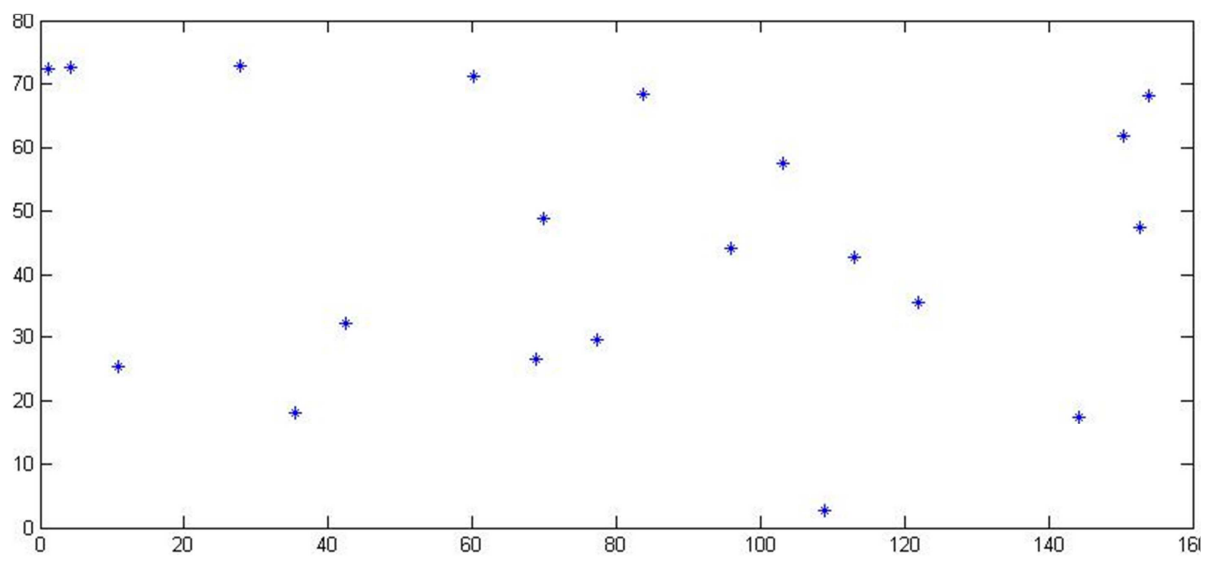

图1 随机生成20个客户的位置分布。

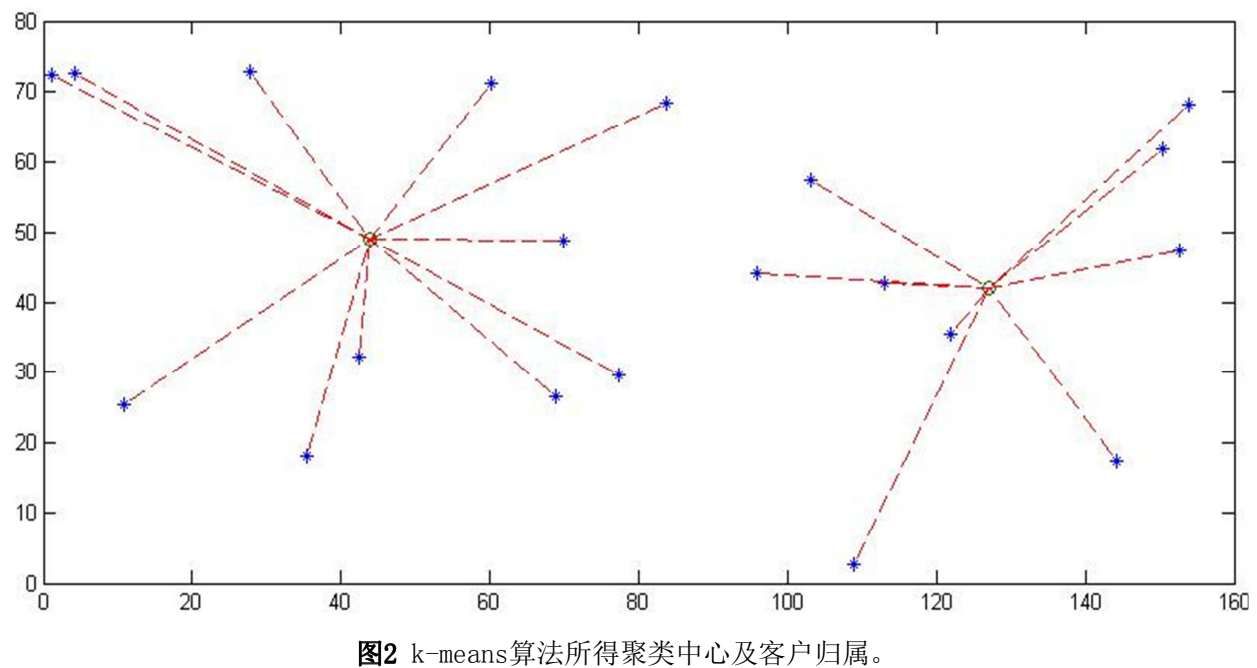


以上步得到的 $k$-means聚类算法结果为例, 使用最小生成树法得到的管道规划图如图3所示:

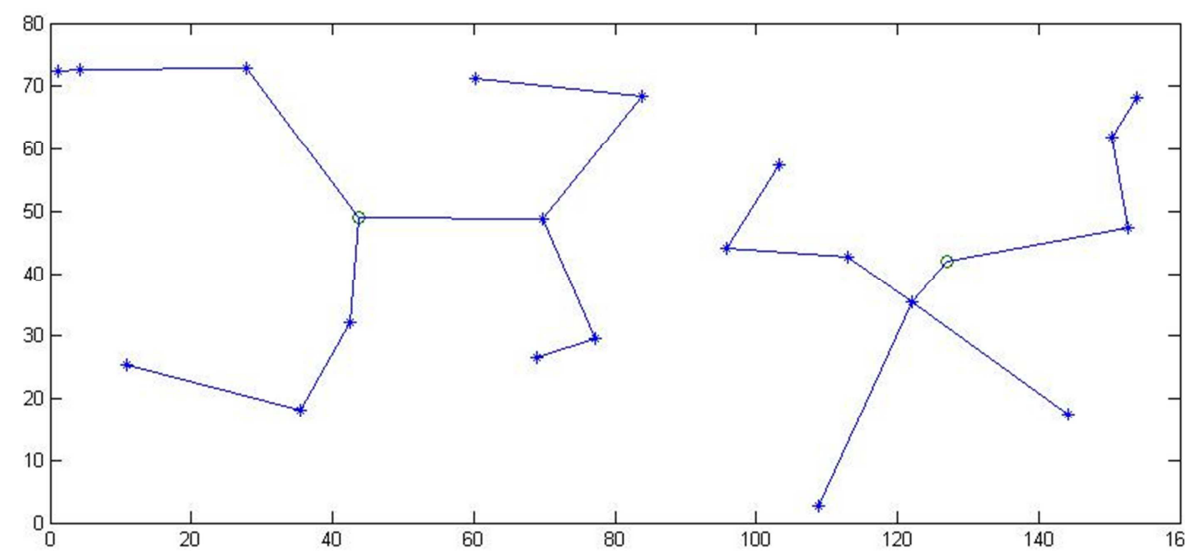

图3 Prim算法求得的管道分布图。

利用上述方法，分别对 $k$ 值取 $1 、 2 、 3$ 和 4 ，根据市场 经验, 模型中的参数取值为: $C_{f}=157424.7 ¥, C_{v f}=268$ $¥ / K W, c_{d}=5805 ¥ / m, c_{o h}=0.24 ¥ / K W h, c_{o p}=0.4$ $Y / K W h$ 。求得最终的目标函数值 $(Y)$ 见表 1 。

表1 不同供应点数目下函数值及运行时间。

\begin{tabular}{lllll}
\hline 供应点K & $\mathbf{1}$ & $\mathbf{2}$ & $\mathbf{3}$ & $\mathbf{4}$ \\
\hline 目标函数值 & 4967911 & 4910822 & 4856791 & 22384376 \\
运行时间 $(\mathrm{s})$ & 1.15 & 1.46 & 1.35 & 0.05 \\
\hline
\end{tabular}

比较结果可知, 此情况下 $k=2$ 时, 可得到目标函数的较优 解, 且其目标函数值远小于 $k=1$ 时的解, 可见能源分散供 应, 梯级利用会大大降低成本。

\subsection{2. 网络中节点的不可运作性}

设此供应链由 $n$ 个节点组成 (用 $1,2,3, \ldots, n$ 表示), 各个 节点之间存在着复杂的相互关系。对于存在相互依存关系 的节点 $j$ 与节点 $i$, 节点 $j$ 对节点 $i$ 的不可运作性如下

$$
q_{i j}=a_{i j} q_{j}
$$

式中, $q_{i j}$ 表示节点 $j$ 对节点 $i$ 通过相互依存关系造成 的不可运作性, $a_{i j}$ 表示节点 $j$ 对节点 $i$ 通过相互依存关系 造成的不可运作性的概率, 满足 $0 \leq a_{i j} \leq 1$, 当 $a_{i j}=0$ 时, 表示节点 $j$ 与节点 $i$ 之间无相互依存关系, 当 $a_{i j}=1$ 时, 表示节点 $j$ 的不可运作必将导致节点 $i$ 的不可运作， $q_{j}$ 表 示节点 $j$ 的不可运作性。

由于节点的不可运作性等于其直接的不可运作性与 其它所有节点对其造成的间接不可运作性的总和, 故有如 下关系式:

$$
q_{i}=\sum_{j=1}^{n} q_{i j}+c_{i j}=\sum_{j=1}^{n} a_{i j} q_{j}+c_{i}, i, j=1,2, \ldots n
$$

式中 $c_{i}$ 表示外界环境对节点 $i$ 造成的直接不可运作性。

$$
A=\left[\begin{array}{cccc}
a_{11} & a_{12} & \cdots & a_{1 n} \\
a_{21} & a_{22} & \cdots & a_{2 n} \\
\vdots & \vdots & \ddots & \vdots \\
a_{n 1} & a_{n 2} & \cdots & a_{n n}
\end{array}\right]
$$

则式(14) 化为:

$$
q=A q+c
$$

\subsection{3. 整体及节点的经济损失}

计算节点的不可运作性的目的, 就是为对整个系统在 突发事件情况下的经济损失提供依据, 为简化计算, 假设 企业的经济损失与不可运作性线性相关。则有如下关系式:

$$
\Delta Q_{i}=q_{i} \cdot Q_{i}, i=1,2, \ldots, n
$$

式中 $Q_{i}$ 表示节点 $i$ 原计划的经济收益, $\Delta Q_{i}$ 表示由于 外界突发性事件导致的节点 $i$ 的经济损失。

$$
\operatorname{diag}(q)=\left[\begin{array}{cccc}
q_{1} & 0 & \cdots & 0 \\
0 & q_{2} & \cdots & 0 \\
\vdots & \vdots & \ddots & \vdots \\
0 & 0 & \cdots & q_{n}
\end{array}\right]
$$

则式(17) 可以用矩阵表示为:

$$
\Delta Q=\operatorname{diag}(q) \times Q
$$

整个分布式能量系统供应链的经济损失 $S$ 表示为:

$$
S=\sum_{i=1}^{n} \Delta Q_{i}=\sum_{i=1}^{n} q_{i} \cdot Q_{i}
$$

\subsection{4. 鲁棒优化策略}

分布式能量系统所面临的问题是不仅仅有其内部的 不确定性, 还有其外部的不可预测性, 面对不安全环境和 不确定因素, 任何不可预测的事件都有可能给整个分布式 
能量系统带来巨大的影响。由于系统各个部分之间相互依 赖, 所以何意节点受到的冲击都有可能沿着系统网络向其 它节点传播, 甚至可能对系统造成毁灭性的破坏。因此, 合理评估分布式能量系统在不安全环境和不确定因素条 件下的性能影响的经济损失, 提高系统对不可预测事件的 鲁棒性在分布式能量系统发展中显得尤为重要 [10]。

输入输出模型 (IIM) 是由Wassily Leontief提出的, Leontief因此项卓越贡献获得了1973的诺贝尔经济学奖 [11]。当风险发生时, 制定较为合理的风险管理策略和 方案。目前在实际应用中的分布式能量系统的鲁棒优化策
略较多, 比如增加系统的库存和增加原材料供应商的数量。 而针对于不同的系统，往往会有不同的优化策略 [12]。

例如图4所示的分布式能量系统, 由于二次供货商只 有一个, 因此二次供应商的破坏必将对整个系统产生很大 的影响, 造成巨大的经济损失, 而另设一个二次供应商之 后, 同样的事件对系统造成的不得影响便会大大降低。

通过计算可得: 优化前节点 3 的破坏, 将导致系统约 126.2 万元的经济损失; 而在优化之后, 节点 3 的破坏将产 生约72.5万元的经济损失。通过鲁棒优化策略, 可使此分 布式能量系统各供应链的运作鲁棒性显著增强。

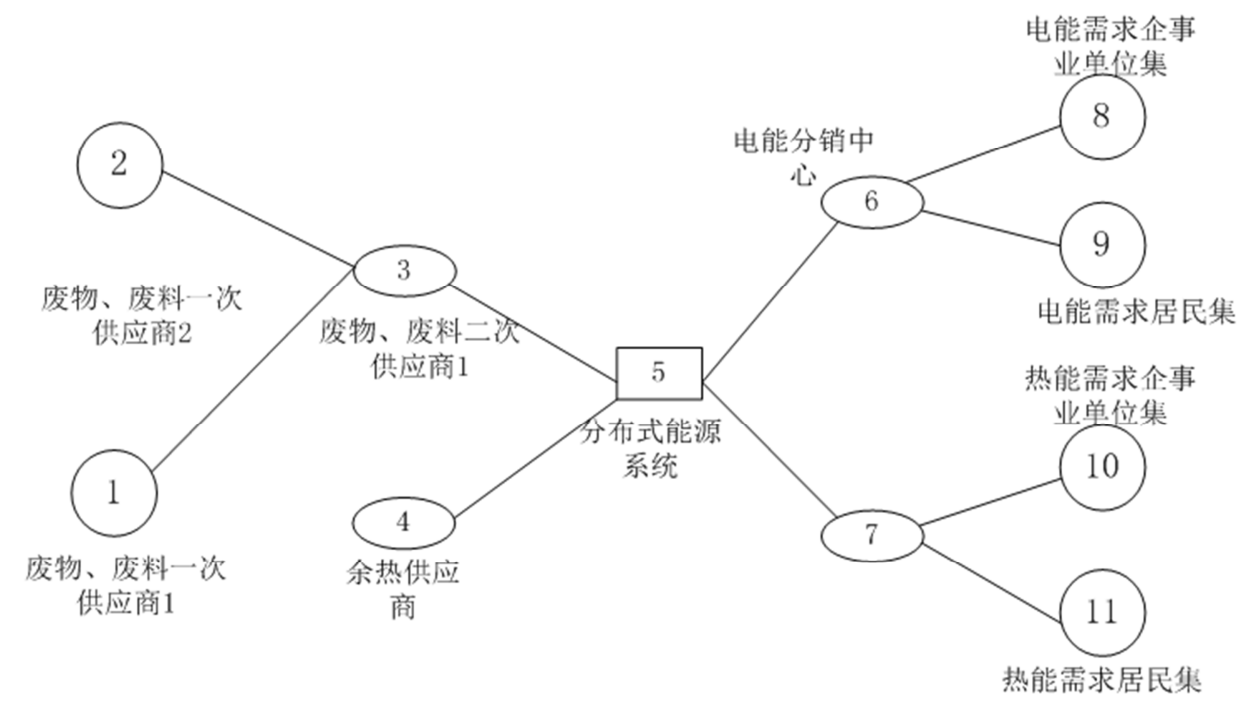

图4 优化前的分布式能量系统供应链结构。

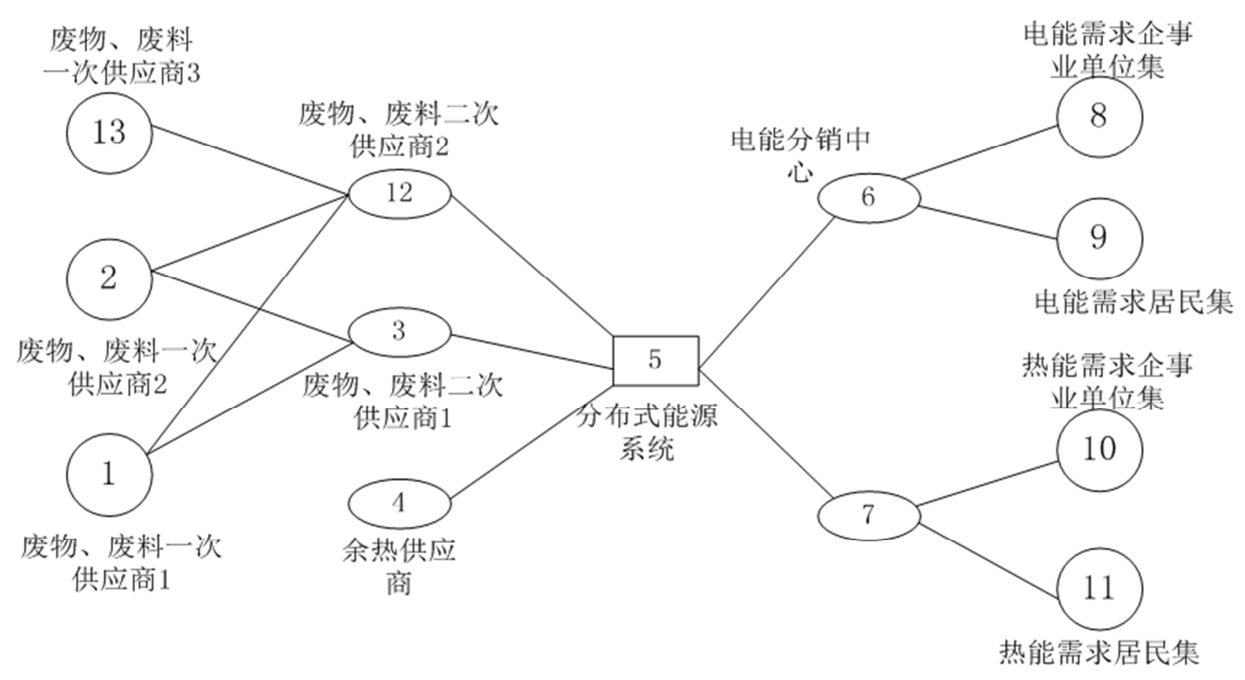

图5 优化后的分布式能量系统供应链结构。

\section{3. 实例计算}

\section{1 . 问题描述}

在某市区中心处现需要对 30 个客户提供能源, 各客户 的坐标及各时段的电能热能需求量如下表所示, 由于在市 区中心, 环境要求较为严格, 并有完善的污染收费标准, 周围原材料供应商充足, 可以灵活选择, 主要污染排放收
费价格为: $C_{N O_{x}}=46.45 Y / k g, C_{C O}=6.26 ¥ / \mathrm{kg}$ 。对 整个供能系统进行规划。

\section{2. 计算分析}

$k=2$ 时供应点的坐标为 S1 为 (104. 200, 41. 467), S2 为 $(25.533,59.000)$, 使用最小生成树, 得到管道归属如 图6所示。 


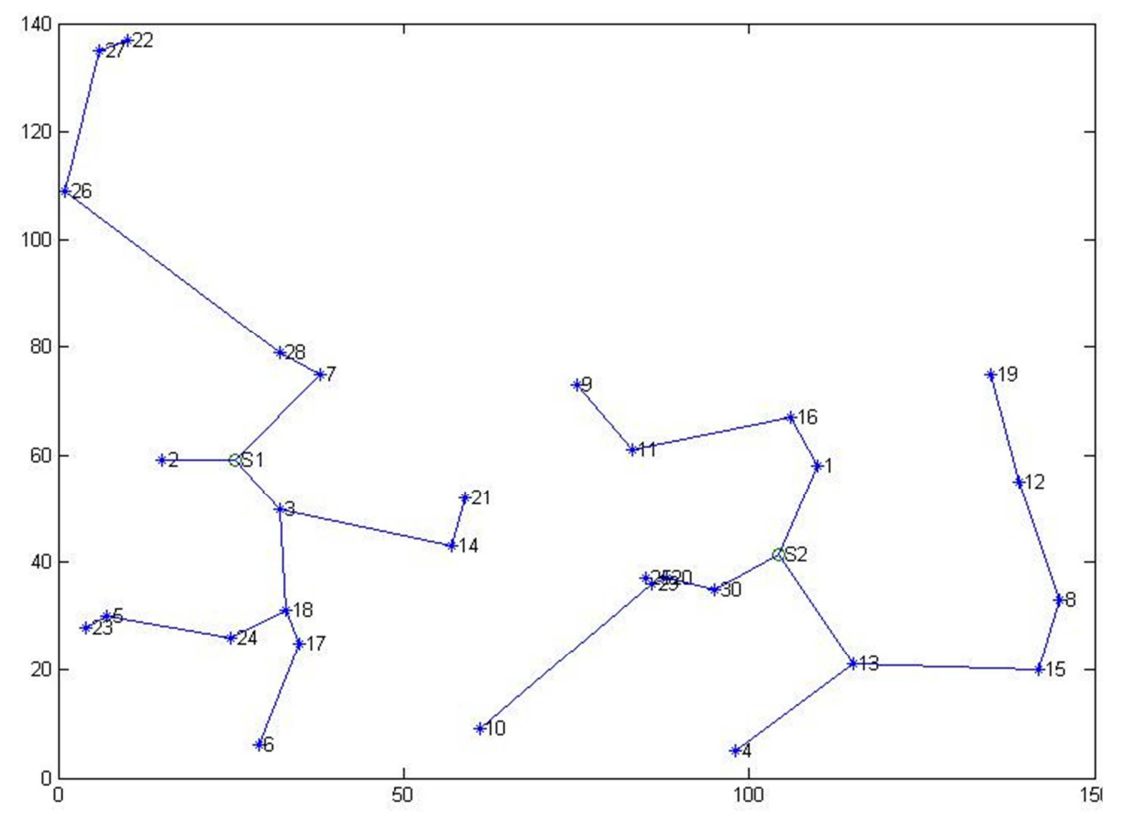

图6 k=2时管道分布示意图。

按照此管道规划, 可得到管道总长度为 $489.71 \mathrm{~m}$, 供 应点 1 的热能产能为 $4545 \mathrm{KWh} / \mathrm{h}$, 电能产能为 4825 $K W h / h$, 供应点 2 的热能产能为 $4493 \mathrm{KWh} / \mathrm{h}$, 电能产能为 $5069 \mathrm{KWh} / \mathrm{h}$ 。

下面考虑污染的处理。使用选择性催化还原技术脱硝 方案, 主要操作参数见表 2 :

根据式 $8^{\sim} 10$, 可求得污染排放量, 分别为 $N O_{x}$ 为 $206.36 \mathrm{~g} / \mathrm{kg}$ fuel $、 C O$ 为 $1007.18 \mathrm{~g} / \mathrm{kg}$ fuel $、 C O_{2}$ 为 $1741.74 \mathrm{~g} / \mathrm{kg}$ fuel, 进而得到污染收费为 $35.17 ¥ / \mathrm{h}$, 目标函数为: $8609268.93 ¥$ 。
表2 主要操作参数。

\begin{tabular}{llll}
\hline 操作参数 & 数值 & 操作参数 & 数值 \\
\hline 烟气与水蒸气的最小温差 $/{ }^{\circ} \mathrm{C}$ & 10 & 透平效率 & 0.990 \\
烟气与过热器夹点温差 $/{ }^{\circ} \mathrm{C}$ & 25 & 蒸气轮机等熵效率 & 0.850 \\
除氧器压力 $/ \mathrm{Mpa}$ & 0.140 & 环境温度 $/{ }^{\circ} \mathrm{C}$ & 15 \\
凝气器压力 $/ \mathrm{Mpa}$ & 0.005 & 㫤机械效率 & 0.900 \\
压缩机的等熵效率 & 0.860 & 年运行时间 $/ \mathrm{h}$ & 8000 \\
发电机效率 & 0.985 & 生活热水温度 $/{ }^{\circ} \mathrm{C}$ & $70^{\sim} 80$ \\
\hline
\end{tabular}

同理求得 $k=3$ 和 $k=4$ 时的目标函数值，分别为: $k=3$ 时, $C_{\text {total }}=8520120.04 ¥, k=4$ 时, $C_{\text {total }}=$ 8935014. $32 Y$, 比较可得, 当 $k=3$ 时, 目标函数可取得 最小值，此时管道分布如下图所示:

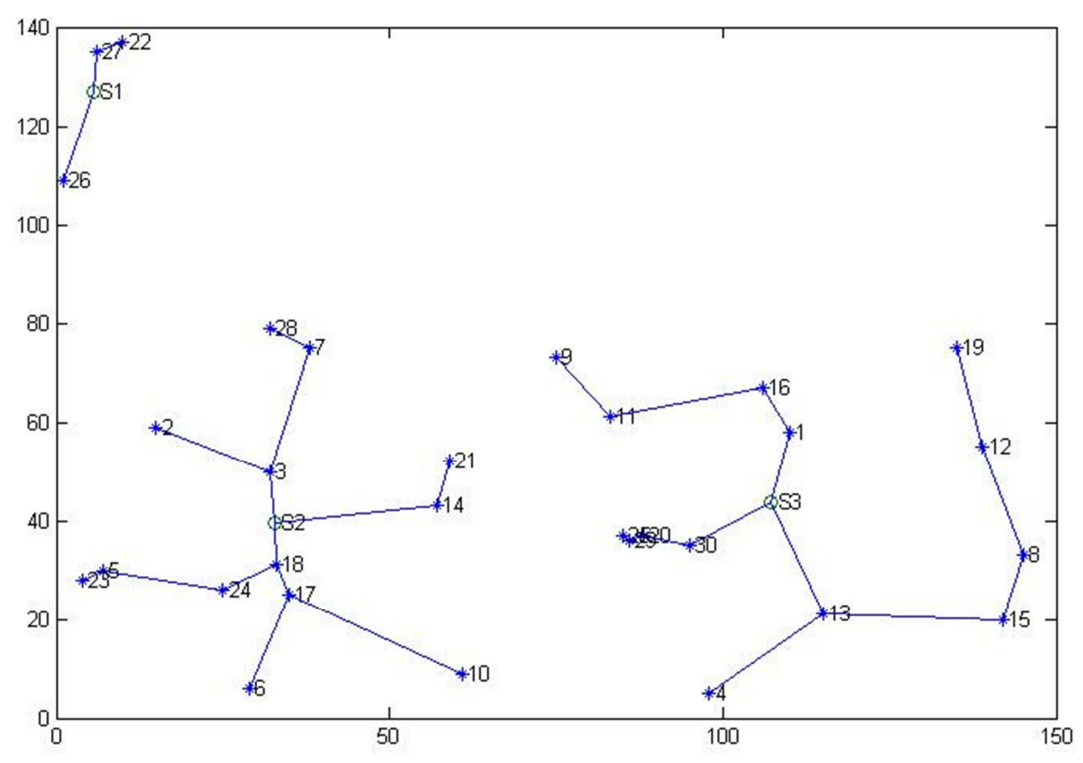

图7 k=3时管道分布示意图。 
下面对其鲁棒性进行研究, 由于原材料供应充足, 供 应链的选择较为灵活, 故可依据图5规划风险削减能源供 应链网络结构.

假设节点3遭到破坏，通过各节点之间的相互关系， 可以得到相互关系矩阵A。

代入式 (19) 和 (20) 中, 可求得:

$Q=\left[\begin{array}{lllllllllllll}63 & 109 & 76 & 33 & 210 & 81 & 64 & 108 & 97 & 116 & 55 & 70 & 58\end{array}\right]^{T}$

供应链的经济损失 (万元) 为:

$$
S=\sum_{i=1}^{6} \Delta Q_{i}=72.493
$$

而若没有加入节点 12 和节点 13 , 则相互关系矩阵当中 应去掉相应节点, 用同样的方法求得：

$$
\begin{gathered}
Q=\left[\begin{array}{lllllllllll}
63 & 109 & 76 & 33 & 210 & 81 & 64 & 108 & 97 & 116 & 55
\end{array}\right]^{T} \\
S=\sum_{i=1}^{6} \Delta Q_{i}=126.219
\end{gathered}
$$

当节点3遭到破坏时，供应链经济损失达到 126.219 万元。可见通过上述优化处理, 系统的鲁棒性能有了很大 的提高。

\section{4. 结论}

本文运用双层规划的相关理论, 以分布式能量系统的 建设和运营所需费用为目标函数, 建立双层规划模型。提 出了 $\mathrm{k}$-means 聚类算法和最小生成树算法求解下层模型, 解决热能运输管道网络的优化问题。通过与传统集中式能 源系统的供应成本相比较, 可发现, 此网络规划有显著的 经济效益。

\section{致谢}

本文为湖北省自然科学基金（No. 2013CFA131）;治金 工业重点实验室基金(Y201411) 资助。

\section{参考文献}

[1] 魏海荵. 分布式能源供应链的规划与鲁棒运作研究 [D]. 上 海交通大学博士学位论文. 2010:1 18

[2] 江泽民. 对中国能源问题的思考 [N]. 力学学报. 2008, 42 (3): $345 \sim 359$

[3] 中国科学院可持续发展研究组. 中国可持续发展战略报告 [C]. 2003. 北京: 科学出版社, 2003:1 2

[4] 吴大为, 王如竹. 分布式能源定义及其与冷热电联产关系的 探讨 [J]. 制冷与空调. 2005, 5(5): 1 6

[5] 骆喆, 丁铭, 罗汉文. 小小区基站网络中能量有效的分布式 动态开关策略 $[\mathrm{J}]$. 上海师范大学学报: 自然科学版 2015, 44 (1) :73 76

[6] 赵珊珊, 宋晓辉, 孟晓丽. 双层次能源联络线结构配电网的 接纳能力分析 $[\mathrm{J}]$. 电力系统自动化, 2016 (2).63 68

[7] 滕春贤, 李智慧. 二层规划的理论与运用 $[\mathrm{J}]$. 科学出版 社. 2002, 3 (2) : 50 60

[8] 张苗. 基于双层规划的多目标校车路径优化研究 [D]. 成都 西南交通大学硕士论文. 2008:2 8

[9] Ulrike von Luxburg. A Tutorial on Spectral Clustering [J]. Statistics and Computing. 2007, $17(4): 395^{\sim} 416$

[10] Frangopoules A. Thermo economic functional analysis optimization [J]. Energy 1987, $12(5): 63^{\sim} 71$

[11] Leontief, W. W. Input-output economics[J]. Scientific American 1951, $185(4): 15^{\sim} 21$

[12] Ulam, S., Richtmyer, R. D., Neumann, J. V. Statistical methods in neutron diffusion[J]. LosAlamos Scientific Laboratory Report. 1947, 3(6):536 ₹551 